\title{
Direct reprogramming of urine-derived cells with inducible MyoD for modeling human muscle disease
}

\author{
Ellis Y. Kim', Patrick Page ${ }^{2}$, Lisa M. Dellefave-Castillo², Elizabeth M. McNally²* and Eugene J. Wyatt ${ }^{2 *}$
}

\begin{abstract}
Background: Cellular models of muscle disease are taking on increasing importance with the large number of genes and mutations implicated in causing myopathies and the concomitant need to test personalized therapies. Developing cell models relies on having an easily obtained source of cells, and if the cells are not derived from muscle itself, a robust reprogramming process is needed. Fibroblasts are a human cell source that works well for the generation of induced pluripotent stem cells, which can then be differentiated into cardiomyocyte lineages, and with less efficiency, skeletal muscle-like lineages. Alternatively, direct reprogramming with the transcription factor MyoD has been used to generate myotubes from cultured human fibroblasts. Although useful, fibroblasts require a skin biopsy to obtain and this can limit their access, especially from pediatric populations.
\end{abstract}

Results: We now demonstrate that direct reprogramming of urine-derived cells is a highly efficient and reproducible process that can be used to establish human myogenic cells. We show that this method can be applied to urine cells derived from normal individuals as well as those with muscle diseases. Furthermore, we show that urine-derived cells can be edited using CRISPR/Cas9 technology.

Conclusions: With progress in understanding the molecular etiology of human muscle diseases, having a readily available, noninvasive source of cells from which to generate muscle-like cells is highly useful.

Keywords: Urine cells, MyoD, Reprogramming, Myotubes, Muscular dystrophy, CRISPR

\section{Background}

The genetic diversity of muscle diseases, including the muscular dystrophies, is extensive $[1,2]$. Defining underlying mechanisms and developing therapies to correct these defects require appropriate models of disease. Animal models offer in vivo insight and have proved highly useful, but animal models are time consuming and costly to develop and cannot represent the large number of alleles responsible for muscle diseases. Cellular models of disease have expanded in recent years with significant advances in reprogramming and the creation of induced pluripotent stem cells (iPSCs) for many disorders. For muscle diseases, the

\footnotetext{
* Correspondence: Elizabeth.mcnally@northwestern.edu; Eugene.wyatt@ northwestern.edu

${ }^{2}$ Center for Genetic Medicine, Northwestern University Feinberg School of Medicine, 303 E. Superior St., Chicago, IL 60611, USA

Full list of author information is available at the end of the article
}

comparative ease of generating cardiomyocytes, rather than skeletal myocytes, from iPSCs has led to this cell type being used as a surrogate for skeletal muscle [3-5]. Although progress has been made in generating muscle-like lineages from iPSCs, this process is still time-consuming and has variable outcomes within and across culture systems [6, 7]. Other commonly used cell models of skeletal muscles include the mouse $\mathrm{C} 2 \mathrm{C} 12$, rat L6, and human myoblast cell lines, which undergo differentiation into multinucleate myotubes under appropriate conditions [8,9]. These myoblast models have been highly useful for the field but represent normal skeletal muscle and not disease-specific conditions. While myoblasts can be cultured from human muscle, biopsies from patients are invasive and at times problematic for those with muscle masslimiting conditions. A noninvasive patient-specific 
in vitro skeletal muscle model is desirable for studying pathogenesis and testing potential drugs.

Direct reprogramming of fibroblasts with MyoD into myotubes was a central experiment used to demonstrate the role of transcription factors $[10,11]$. Since that original observation, MyoD delivery into fibroblasts has been optimized using viral vectors and this method can be applied to make directly reprogrammed myogenic cells from humans and mice [12, 13]. A fusion between MyoD and the estrogen receptor was generated to create a tamoxifen-inducible MyoD, and this construct was used in mouse and human fibroblasts to induce myogenesis [14-16]. Thus, direct reprogramming of fibroblasts remains a highly useful method for modeling human muscle diseases. However, dermal fibroblasts are obtained through skin biopsy, and the invasive nature of skin biopsy, while considerably less than muscle biopsy, can be particularly problematic in children.

One potential noninvasive source of patient-specific cells is cells isolated from urine. It has been estimated that about 2000 to 7000 renal tubular epithelial cells are shed in urine everyday $[17,18]$. Obtaining a urine sample is noninvasive, making urine a good source of cells, especially from children. Urine cells have been isolated from both healthy volunteers and patients of a wide age range [18-21]. Urine-derived cells (UDCs) are thought to be of a mixed population that originates from either the renal epithelium or the uroepithelium [18, 21]. Interestingly, a subset of UDCs has been observed to carry mesenchymal stem cell markers as well as low endogenous expression of Oct3/4, a pluripotency marker [4, 20, 22]. As such, UDCs demonstrate propensity for differentiation into several lineages, one of which is muscle-like [20, 23].

We show here that UDCs serve as a noninvasive source for MyoD-induced myogenesis. UDCs transduced with a tamoxifen-inducible MyoD lentiviral vector (iMyoD) formed multinucleate myotubes following induction with tamoxifen and culture in differentiation media for up to 35 days. Urine cell-derived myotubes expressed muscle marker transcripts, formed sarcomeres, and displayed contractile properties. Further, UDCs isolated from individuals with muscular dystrophies were also able to form myotube-like structures and reflected the disease mutations. Thus, UDCs are a ready source for direct reprogramming by MyoD and provide an easily obtainable, noninvasive source for modeling disease mechanisms.

\section{Methods}

\section{Urine collection and cell isolation}

Written and informed consent was obtained from all human subjects. All work was approved by and conducted under the Institutional Review Boards of the
University of Chicago (IRB8249) and Northwestern University (IRB STU00104548), which serve as the ethics boards for each institution, respectively. All studies were conducted in compliance with the Helsinki Declaration. Urine samples were collected using midstream clean catch kits according to the directions provided in the kits (Parter Medical Products Inc, Carson, CA; 273516). Cells were isolated within $1 \mathrm{~h}$ of collection using a protocol previously published with minor modifications [24]. Briefly, the entire urine samples were equally distributed into 50$\mathrm{mL}$ conical tubes and centrifuged at $400 \times g$ for $10 \mathrm{~min}$ at room temperature. The supernatant was aspirated leaving $\sim 1 \mathrm{~mL}$ of urine into which pellets were resuspended and combined into a single tube, if necessary. Ten milliliters of wash buffer was added per $100 \mathrm{~mL}$ of initial urine sample. Samples were centrifuged at $200 \times g$ for $10 \mathrm{~min}$ at room temperature. The supernatant was aspirated leaving $\sim 0.2 \mathrm{~mL}$, and the cell pellet was resuspended in $1 \mathrm{~mL}$ of primary media. All media formulations were obtained from a previously published protocol and are detailed below [24]. Cells were plated in 24-well plates pre-coated with $0.1 \%$ gelatin (Millipore, Billerica, MA; ES-006-B, Stemcell Technologies, Vancouver, Canada; 7903). Roughly one third of the cell suspension was plated in the first well, with the remaining two thirds equally divided into four additional wells. The final volume in each well was then brought to $500 \mu \mathrm{L}$ with primary media. The plates were placed in a $37^{\circ} \mathrm{C}$ incubator with $5 \% \mathrm{CO}_{2}$.

For 3 days, $500 \mu \mathrm{L}$ of primary media was added to each well every $24 \mathrm{~h}$. On day $4,1.5 \mathrm{~mL}$ of primary media was removed and replaced with $500 \mu \mathrm{L}$ of proliferation media. An aliquot of the primary media was added to a separate dish containing Dulbecco's Modified Eagle Medium (DMEM) supplemented with $10 \%$ FBS without antibiotics or antimycotics to test for potential contamination. On day 5 , all media were removed from each well and replaced with $500 \mu \mathrm{L}$ of proliferation media, which was changed daily until the isolated cells expanded and were replated in larger dishes. Antibiotics and antimycotics were removed from media once uncontaminated cultures were confirmed. Isolated cells were observed as early as 1 day after the addition of proliferation media. When the cells became confluent or when cell foci began to outgrow the monolayer, cells were trypsinized using $0.25 \%$ trypsin-EDTA (Thermo Fisher Scientific, Waltham, MA; 25200-072), subcultured, and designated as passage 1 (p1). Modifications from [24] include plating of cells in five wells of a 24-well gelatin-coated plate (vs a single well of 12-well plate), increase of FBS content in the proliferation media to $15 \%$, and the removal of the antimycotics and 
antibiotics from the media after lack of contamination was observed.

\section{Media composition}

All media were made following a previously published protocol with the following modifications [24]. Wash buffer consisted of $1 \times$ phosphate-buffered saline (PBS) without $\mathrm{Ca}^{2+}$ and $\mathrm{Mg}^{2+}$ (Thermo Fisher Scientific, Waltham, MA; 14190-250) supplemented with $1 \%$ penicillin/streptomycin (Thermo Fisher Scientific, Waltham, MA; 15070-063) and $0.5 \mu \mathrm{g} / \mathrm{mL}$ amphotericin B (Sigma Aldrich, St. Louis, MO; A2942). Primary media were composed of 1:1 mix of high glucose DMEM without sodium pyruvate (GE Healthcare, Logan, UT; SH30022.FS) and Ham's F-12 Nutrient Mix (Thermo Fisher Scientific, Waltham MA; 11765-054) supplemented with Renal Epithelium Growth Medium SingleQuot Kit Supplements (Lonza, Basel, Switzerland; CC-4127), $10 \%$ fetal bovine serum (Thermo Fisher Scientific, Waltham, MA; 16000-044), $1 \%$ penicillin/ streptomycin, and $0.5 \mu \mathrm{g} / \mathrm{mL}$ amphotericin B. Proliferation media were composed of 1:1 mix of Renal Epithelium Growth Medium Bullet Kit (Lonza, Basel, Switzerland; CC-3190) and high glucose DMEM supplemented with 15 \% FBS, 0.5 \% Glutamax (Thermo Fisher Scientific, Waltham, MA; 35050-061), 0.5 \% nonessential amino acids (Thermo Fisher Scientific, Waltham, MA; 11140-050), and $2.5 \mathrm{ng} / \mathrm{mL}$ of bFGF (Peprotech, Rocky Hill, NJ; 100-18B, Miltenyi Biotec Inc, San Diego, CA; 130-093-842), PDGF-AB (Peprotech, Rocky Hill, NJ; 100-00AB), and EGF (Peprotech, Rocky Hill, NJ; AF-100-15). The Renal Epithelium Growth Medium (REGM) Bullet Kit was made according to the manufacturer's instructions, with the omission of the amphotericin B/gentamycin supplement. Freeze media were composed of DMEM (Thermo Fisher Scientific, Waltham, MA; 11995-073) supplemented with $30 \%$ FBS, 1x pen/strep, and 10 \% DMSO (Sigma Aldrich, St. Louis, MO; D2650).

\section{Lentiviral construct and transduction}

The tamoxifen-inducible MyoD lentiviral construct (pLv-CMV-MyoD-ER(T)), referred to as iMyoD, was previously described [14] and kindly provided by Dr. Jeffrey Chamberlain (University of Washington; Addgene plasmid \# 26809). The construct was packaged by the Skin Disease Research Center DNA/RNA Delivery Core at Northwestern University (Chicago, IL). Urine cells from healthy volunteers or patients were plated in 12-well plates at 75,000 cells/well and transduced within 24-36 h of plating with iMyoD at MOI $=50$. Transductions were performed in proliferation media with reduced serum (5\%) and $7.5 \mu \mathrm{g} / \mathrm{mL}$ polybrene (Millipore, Billerica, MA; TR-1003-G) for 6-16 h in a $37^{\circ} \mathrm{C}$ incubator.

\section{Evaluation of transduction efficiency}

After transduction, an aliquot of cells were plated in two wells of a 24-well plate at 2000-10000 cells $/ \mathrm{cm}^{2}$. Cells were treated with $5 \mu \mathrm{M}$ 4-hydroxytamoxifen (Sigma Aldrich, St. Louis, MO; H7904) for 24 to 48 h, and MyoD was detected using immunofluorescence. Methods and antibodies are as described below. A minimum of three fields and a minimum of 100 cells total were analyzed to determine transduction efficiency. MyoD-positive nuclei and total nuclei were counted per field, and efficiency was calculated by dividing the number of MyoD-positive nuclei by the total number of nuclei per field. Efficiencies were graphed as mean \pm standard error of the mean (SEM) using GraphPad Prism (GraphPad, La Jolla, CA). All images were taken using the Floid Cell Imaging Station (Thermo Fisher Scientific, Waltham, MA; 4471136). The cell counts for MyoD-positive nuclei were performed manually using ImageJ (NIH, Bethesda, MD), while the cell counts for total nuclei were performed using the Particle Analyzer function of ImageJ (NIH, Bethesda, $\mathrm{MD)}$.

\section{Myogenic differentiation}

Transduced cells were plated in 24-well plates coated with $8 \mu \mathrm{g} / \mathrm{cm}^{2}$ collagen I (Sigma Aldrich, St. Louis, MO; C3867, Corning, Corning, NY; 354236) at a density of 37,500 to 50,000 cells $/ \mathrm{cm}^{2}$ and cultured until confluent. Cells were then overlaid with Growth Factor Reduced Matrigel (Corning, Corning, NY; 354230) diluted 1:4 in cold DMEM/F-12 50:50 mix (Corning, Corning, NY; 10-092-CV). Matrigel was allowed to gel for 2 to 3 hours at $37{ }^{\circ} \mathrm{C}$. The Matrigel-overlaid iMyoD-treated cells were induced with 4-hydroxytamoxifen (2.5 $\mu \mathrm{M}, 24-$ $48 \mathrm{~h}$ ) diluted in high glucose DMEM (Thermo Fisher Scientific, Waltham, MA; 11995-073) supplemented with $10 \%$ FBS. Hydrocortisone dexamethasone (HD) differentiation media were based on previously published formulation [23, 25] and were composed of high glucose DMEM with sodium pyruvate supplemented with 10 \% FBS, 5 \% horse serum (Thermo Fisher Scientific, Waltham, MA; 16050-122), $50 \mu \mathrm{M}$ hydrocortisone (Sigma Aldrich, St. Louis, MO; H0888), and $0.1 \mu \mathrm{M}$ dexamethasone (Sigma Aldrich, St. Louis, MO; D4902). Two hundred and fifty microliters of differentiation media was replaced every $72 \mathrm{~h}$. Additional coatings tested during plating optimization included collagen IV (Sigma Aldrich, St. Louis, MO; C5533), fibronectin (Sigma Aldrich, St. Louis, MO; F1141), laminin (Sigma Aldrich, St. Louis, MO; L2020), Growth Factor Reduced Matrigel, poly-L-lysine (Sigma Aldrich, St. Louis, MO; 
P4832), and poly-D-lysine (Sigma Aldrich, St. Louis, MO; P6407).

\section{RNA analysis}

Differentiated myotubes were collected at three different time points (7, 14, and 28 days). Cells were gently washed with cold $1 \times$ PBS. Washes were repeated until most of the Matrigel was removed. RNA was isolated in TRIzol (Thermo Fisher Scientific, Waltham, MA; 15596018) following the manufacturer's instructions. Glycogen (Thermo Fisher Scientific, Waltham, MA; AM9510) was added at $50-100 \mu \mathrm{g} / \mathrm{mL}$ to the isopropanol before RNA precipitation. RNA concentration was determined using NanoDrop 2000 (Thermo Fisher Scientific, Waltham, MA). RNA (1000-1500 ng) was reverse transcribed using qScript cDNA SuperMix (Quanta Biosciences, Gaithersburg, MD; 95048-025). Complementary DNA (cDNA) (30-50 ng) was used per PCR reaction, and the products were separated on $1.5 \%$ agarose gel with ethidium bromide. Gels were visualized and imaged on the UVP Transilluminator (UVP, Upland, CA). Quantitative PCR was performed with 30-50 ng of cDNA using iTaq Universal SYBR Green Supermix (Bio-Rad, Hercules, CA; 1725124) with biological triplicates when available and technical duplicates. $\mathrm{Cq}$ values were averaged for each pair of technical duplicates and normalized to GAPDH. $\triangle \mathrm{Cq}$ values were calculated as $\mathrm{Cq}_{\text {(gene of interest) }}-\mathrm{Cq}_{(\mathrm{GAPDH})}$, and $\Delta \Delta \mathrm{Cq}$ values were calculated as $2^{\wedge}(-\Delta \mathrm{Cq}) . \Delta \Delta \mathrm{Cq}$ values were then multiplied by 1000 as an arbitrary factor for graphing purposes. $\Delta \Delta \mathrm{Cq}$ values were graphed as mean $\pm \mathrm{SEM}$ using GraphPad Prism (GraphPad, La Jolla, CA). Primers used for RT- and qRT-PCR are listed in Tables 1 and 2 .

\section{Immunofluorescence microscopy}

Differentiated myotubes were subject to immunofluorescence microscopy at four different time points $(7,14,28$, and 35 days). Myotubes were gently washed with $1 \times$ PBS (Thermo Fisher Scientific, Waltham, MA; 14200-166) three times, or until most of the Matrigel was removed, and then fixed in either $4 \%$ paraformaldehyde (15 min at RT) or ice cold $100 \%$ methanol ( 2 min on ice). Myotubes fixed in paraformaldehyde were subsequently permeabilized in $0.25 \%$ Triton-X (Sigma Aldrich, St. Louis, MO; T8787) for $20 \mathrm{~min}$ at RT. Cells were blocked in $10 \%$ horse serum for $30 \mathrm{~min}$ to $1 \mathrm{~h}$ at $4{ }^{\circ} \mathrm{C}$. Primary antibody incubations were done overnight at $4{ }^{\circ} \mathrm{C}$. Cells were washed three times for $10 \mathrm{~min}$ each with PBS, where PBS was supplemented with $0.1 \%$ Triton-X in the second wash. Cells were incubated with secondary antibodies ( $1 \mathrm{~h}$ at $\mathrm{RT}$ ) and then washed three times for $10 \mathrm{~min}$ each as described above. Nuclei were stained with Hoechst 33342 (1:10,000, Thermo Fisher Scientific,
Table 1 Primers for PCR

\begin{tabular}{|c|c|}
\hline \multirow[t]{2}{*}{ ACTN2 } & F: CTAAAATGTTGGATGCTGAAGACA \\
\hline & R: CAGCAATATCCGACACCATCTTGC \\
\hline \multirow[t]{2}{*}{ DES } & F: AAGGGCACTAACGATTCCCT \\
\hline & R: CATCCCGTGTCTCGATGGTC \\
\hline \multirow[t]{2}{*}{ DMD (exons 1 to 3 ) } & F: TCCTGGCATCAGTTACTGTGTT \\
\hline & R: TATGCTGCTTCCCAAACTTAGAA \\
\hline \multirow[t]{2}{*}{ DMD (exons 42 to $50 / 51$ ) } & F: CAATGCTCCTGACCTCTGTG \\
\hline & R: GAGTAGGAGAGGCTCCAATA \\
\hline \multirow[t]{2}{*}{ DMD (exons 44 to 48 ) } & F: GAACAGTTTCTCAGAAAGAC \\
\hline & R: GCAGCAGATGATTTAACTGC \\
\hline \multirow[t]{2}{*}{ GAPDH } & F: ACCACAGTCCATGCCATCAC \\
\hline & R: CCACCACCCTGTTGCTGTAG \\
\hline \multirow[t]{2}{*}{ MYH2 } & F: GAGGCTGACTCGTCCTGCTTTA \\
\hline & R: GACTGATTCTCTCGGTCAGTCA \\
\hline \multirow[t]{2}{*}{ MYH3 } & F: CTTGTGGGCGGAGGTCTGG \\
\hline & R: GCCACTTGTAGGGGTTGACA \\
\hline \multirow[t]{2}{*}{ MYOG } & F: ACCCAGGGGATCATCTGCTCA \\
\hline & R: CACTGGCATCGGGAAGAGAC \\
\hline \multirow[t]{2}{*}{ SGCG } & F: TCTAAGATGGTGCGTGAGCAG \\
\hline & R: GCCACAGACAGGTACAGCTT \\
\hline
\end{tabular}

Waltham, MA; H3570). Plates were imaged on the Zeiss Axio Observer Z.1 inverted microscope (Carl Zeiss Microscopy, Jena, Germany) and/or Floid Cell Imaging Station. All antibodies were diluted in $0.1 \%$ Triton-X and $2 \%$ horse serum, and all solutions were made with $1 \times$ PBS.

\section{Antibodies}

Primary antibodies were as follows: rabbit polyclonal anti-MyoD (1:1000, Santa Cruz, Dallas, TX; sc-304), mouse monoclonal anti-desmin (1: 1000, Sigma Aldrich, St. Louis, MO; D1033), mouse monoclonal anti- $\alpha$ -

Table 2 Primers for $\mathrm{qPCR}$

\begin{tabular}{ll}
\hline ACTN2 & F: CTAAAATGTTGGATGCTGAAGACA \\
RES & R: CATTCCAAAAGCTCACTCGCTA \\
& F: GATCAATCTCCCCATCCAGA \\
R: TGGCAGAGGGTCTCTGTCTT & F: GTGGACCTGACCTGCCGTCT \\
RAPDH & F: GGAGGAGTGGGTGTCGCTGT \\
MYH2 & R: TGCGCAGTCAGGTCATTGAT \\
& F: TTGATGCCAAGACGTATTGCT \\
MYH3 & R: GGGGGTTCATGGCGTACAC \\
MYOG & F: GCTGTATGAGACATCCCCCTA \\
& R: CGACTTCCTCTTACACACCTTAC \\
\hline
\end{tabular}


actinin (1: 1000, Sigma Aldrich, St. Louis, MO; A7811), rabbit polyclonal anti-dystrophin (1: 1000, Thermo Fisher Scientific, Waltham, MA; PA1-37587), mouse monoclonal anti-dystrophin (1: 100, Leica Biosystems, Newcastle, UK; NCL-DYSB), mouse monoclonal antiMYH1 (1: 10 or $2 \mu \mathrm{g} / \mathrm{mL}$, deposited to Developmental Studies Hybridoma Bank by Fischman, DA; MF 20), mouse monoclonal anti-fast myosin heavy chain (1: 1000, Sigma Aldrich, St. Louis, MO; M4276), mouse monoclonal anti-titin antibody (1: 10 or $2 \mu \mathrm{g} / \mathrm{mL}$, deposited to Developmental Studies Hybridoma Bank by Greaser, ML; 9 D10), and rabbit polyclonal anti- $\gamma-$ sarcoglycan (1: 300 to 1: 500 [26]). Secondary antibodies were as follows: Alexa Fluor 488 donkey anti-rabbit (1: 1,1000; A21206), Alexa Fluor 488 donkey anti-mouse (1: 1000; A21202), Alexa Fluor 594 donkey anti-rabbit (1: 1000; A21207), and Alexa Fluor 594 donkey anti-mouse (1: 1,1000; A21203, all Thermo Fisher Scientific, Waltham, MA).

\section{Sequencing}

$D M D$ exons 42 through the junction of exons 50 and 51 were amplified by PCR, gel purified using QIAEXII Gel Extraction Kit (Qiagen, Valencia, CA; 20021). The purified products were amplified by PCR using nested primers for $D M D$ exons 44 through 48 and then sequenced at the NUSeq Core at Northwestern University (Chicago, IL). Chromatograms and sequences were analyzed on SeqMan Pro (DNASTAR, Madison, WI) and FinchTV (Geospiza, Seattle, WA).

\section{CRISPR/Cas9 design}

To generate a point mutation in control urine cells, we used the Cas9-GFP plasmid designed by the Zhang lab (Addgene plasmid \#48138) [27]. The CRISPR design tool (crispr.mit.edu) was used to identify and select guide RNA (gRNA) targets in human SGCG exon 6. The target on the anti-sense strand was gRNA 5' CTAAGGTCTT GAAACGGGT 3'. This guide permitted a synonymous mutation of the protospacer adjacent motif (PAM) and preserved the 5' SGCG exon 6 sequence. The gRNA was expressed from the U6 polymerase III promoter as previously described [28]. The promoter, crRNA-tracrRNA, and target were synthesized as a gBlock by Integrated DNA Technologies (IDT, Coralville, IA), then PCR amplified (F: TGTACAAAAAAGCAGGCTTTAAAG; R: TAATGCCAACTTTGTACAAGAAAG), and purified using the Qiagen PCR purification kit (Qiagen, Valencia, CA; 28106). The single-stranded oligodeoxynucleotide (ssODN) was designed according to published guidelines [29]. The ssODN, based on the anti-sense strand template, was centered in between the nuclease site and the desired thymine deletion with 71 bp flanking arms on either side (142 bp total). It contained a downstream $\mathrm{G}>\mathrm{A}$ synonymous mutation that destroyed the PAM -NGG site (CGG > CAG). The ssODN ultramer ggagaggttgttaatatttgaacaaaaattct tacCTAAGGTCTTGAAACGGGTCaGCTCTGACAAGGG GTGTCTCCACTGAATGT TCAAAAGAGCCCCTTCAG GCCctaaacaaaaaacaaagatgtatcaggagcagaag was synthesized and PAGE purified by IDT. Uppercase letters indicate the SGCG exon 6 coding region, and lowercase letters indicate the flanking intronic regions. The single underlined region encodes the targeted mutation of the protospacer adjacent motif (PAM), and the italicized sequence is the 4 adenine (AAAA) sequence which encodes the single thymine (T) deletion in the exon 6 coding region.

\section{Electroporation with CRISPR reagents}

On the day of electroporation, urine cells were trypsinized, centrifuged at $400 \times g$ for $5 \mathrm{~min}$ (RT), and resuspended in PBS. An aliquot was removed for cell counting, and cells were centrifuged at $400 \times g$ for $5 \mathrm{~min}$ (RT). Cells were resuspended in electroporation buffer $1 \times 10^{6}$ cells $/ \mathrm{mL}$ (Bio-Rad, Hercules, CA; 165-2677) and $2 \times 10^{5}$ cells were added to the Cas9 GFP plasmid $(1 \mu \mathrm{g})$, U6 gRNA (500 ng), and ssODN (0.5 nmol) reagents. After gentle mixing, the cells were placed in a sterile cuvette with a 0.4-cm gap (Bio-Rad, Hercules, CA; 1652088) and electroporated using Gene Pulser Xcell Electroporation System (Bio-Rad, Hercules, CA; 1652660) with the following settings: (1) single square wave pulse, $200 \mathrm{~V}, 20 \mathrm{msec}$ or (2) three square waves pulses, $150 \mathrm{~V}$, $20 \mathrm{msec}$ ( $0.1 \mathrm{~s}$ between pulses). The electroporated cells were added to gelatin-coated six-well plates and brought to a final volume of $2 \mathrm{~mL}$ with proliferation media. The media were replaced within $12 \mathrm{~h}$ and replaced every $24 \mathrm{~h}$ for 3-5 days until fluorescence-activated cells sorting (FACS).

\section{FACS sorting and clonal expansion}

Prior to cell sorting, 96-well plates were pre-coated with $0.1 \%$ gelatin and placed in the incubator for $1 \mathrm{~h}$. Gelatin was removed and replaced with $100 \mu \mathrm{L}$ of proliferation media that were supplemented with $0.5 \times$ pen/strep. The electroporated cells were trypsinized, centrifuged, and resuspended in $1 \mathrm{~mL}$ of proliferation media supplemented with $0.5 \times$ pen/strep. Single cell sorting of GFPpositive cells into 96 -well plates was conducted by the Northwestern Flow Cytometry Facility using the FACS ArialI (BD Biosciences, San Jose, CA). After sorting, 96-well plates were briefly centrifuged at $200 \times g$ for $1 \mathrm{~min}$ (RT) and placed in a $37{ }^{\circ} \mathrm{C}$ incubator. After $48 \mathrm{~h}, 100 \mu \mathrm{L}$ of proliferation media without pen/strep was added to the 96-well plates. A complete media exchange was performed on days 4, 8, and 12 using proliferation media without pen/strep. Expansion of single cell clones was evident 7-10 days after FACS, with confluent wells observed about day 14 . 
Confluent wells were subcultured onto gelatin-coated 24-well plates until confluent and then subcultured onto gelatin-coated six-well plates.

\section{Genotype analysis of clones}

Clones expanded to the six-well stage were analyzed for genome editing via the CRISPR/Cas9 system. Genomic DNA was isolated and PCR amplified using the GeneArt cleavage detection kit (Thermo Fisher Scientific, Waltham, MA; A24372) according to the manufacturers' instructions. The primer set amplified a genomic region containing SGCG exon 6 along with a portion of the flanking introns (F: TAGGGAAAGAGTTGGGCCTATTT) (R: GAGGCAGATTGGAGAGGAAGAA). To test for clones edited through homology-dependent repair (HDR), PCR-amplified products were subjected to Alu1 digest and restriction fragment length polymorphism (RFLP) analysis. In addition, PCR products from individual clones were sequenced at the NUSeq Core at Northwestern University (Chicago, IL). Chromatograms and sequences were analyzed using SeqMan Pro (DNASTAR, Madison, WI).

\section{Results \\ Generation of urine-derived cells with inducible MyoD expression}

Urine-derived cells were previously shown to be a useful source for the generation of iPSCs [30]. To test direct reprogramming into myogenic lineages using urine cells, we used viral delivery of the muscle transcription factor MyoD. We used a previously characterized tamoxifeninducible MyoD (iMyoD) lentivirus, which permits the culture and expansion of cells in an undifferentiated state until a reprogramming start point is determined [14]. The procedure includes urine cell isolation, proliferation, $\mathrm{iMyoD}$ transduction, tamoxifen induction, myogenic differentiation, and evaluation (Fig. 1a). Urine was isolated from multiple healthy individuals $(n>8)$, and urine cells were isolated and cultured as described $[22,24]$. Bright-field images from two typical urine cultures demonstrated characteristic urine cell morphology and growth (Fig. 1b). More than one cellular morphology was noted, and independent cultures from the same individual were observed to have differential growth rates. In general, we selected clones demonstrating rapid expansion and displaying a type 1 morphology, characterized as a monolayer of polar cells with a "rice-grain" appearance, that maintained close cell-cell contact $[19,31,32]$. Both fresh and freeze-thawed urine cells were used for lentiviral transduction with success.

Urine cells were transduced with the iMyoD lentivirus, regularly achieving $>80 \%$ efficiency with an MOI of 50 , as determined by immunofluorescence microscopy and image analysis for MyoD expression (Fig. 1c, d). In the absence of tamoxifen, MyoD was seen in cytoplasm. After tamoxifen exposure, MyoD staining also became nuclear (Fig. 1c). Because lentiviral transduction was of such high efficiency, nuclear MyoD staining was routinely evident in $80 \%$ of the cultures (Fig. 1d).

\section{Morphological and molecular myogenic reprogramming of iMyoD urine-derived cells}

After iMyoD transduction, urine cells underwent testing for myogenic reprogramming with or without tamoxifen followed by culture in differentiation media (HD media), a formulation previously shown to enhance the endogenous myogenic potential of untransduced urine cells [23]. RT-PCR amplification showed upregulation of the DES (desmin) transcript in tamoxifen-treated cells by the 7-day (7d) time point, and this was sustained throughout the differentiation time course (Fig. 2a). Low-level DES expression was detected in uninduced iMyoD urine cells cultured in HD media alone (Fig. 2a, b). Immunofluorescence microscopy showed that desmin protein expression was evident in tamoxifen-induced iMyoD cells at the earliest time point $(7 \mathrm{~d})$ and increased with extended culture in HD differentiation media (Fig. 2c, d). Desmin protein expression was observed concomitant with increased myotube organization and elongation (Fig. 2c, d). Desmin protein expression was not readily evident in uninduced iMyoD cells (Fig. 2c).

\section{Myogenic differentiation and maturation}

We assessed myotube differentiation on alternative coated surfaces including collagen, laminin, fibronectin, or poly-lysine. We also assessed the effectiveness of Matrigel overlay. We found that matrigel overlay of cells on collagen I-treated plates prior to tamoxifen induction offered the most robust differentiation into myotubes based on morphological appearance. Matrigel overlay was applied when cells reached confluence, and this was followed by tamoxifen induction in DMEM with $10 \%$ FBS $(2.5 \mu \mathrm{M}, 24-48 \mu)$, and culture in HD media for the indicated time course. The outline for this optimized protocol is depicted in Fig. 3a.

MyoD controls temporal expression of early and late genes in muscle development through a feed-forward mechanism [33]. To characterize myogenic marker expression, we tested tamoxifen-treated iMyoD cells by RT-PCR. Robust and sustained expression of MYOG (myogenin) and MYH3 (embryonic myosin) was observed (Fig. 3b). In addition, there was a temporal increase in expression of ACTN2 ( $\alpha$-actinin) and $D M D$ (dystrophin) (Fig. 3b). Expression of $M Y H 2$, the adult 
a

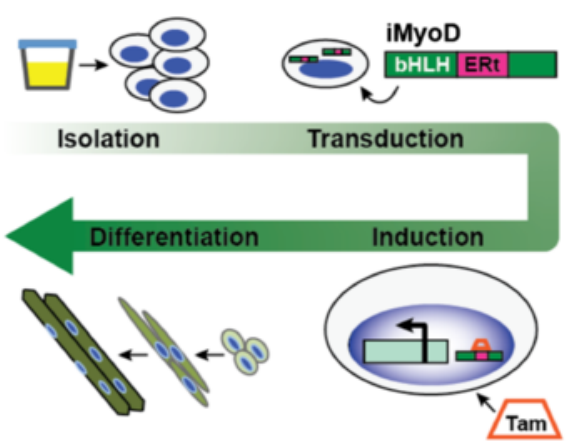

C
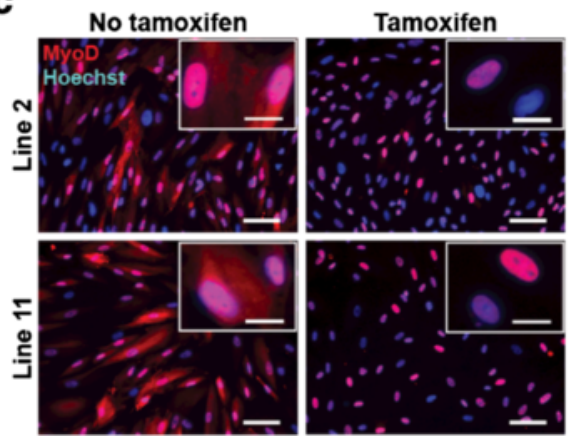

b

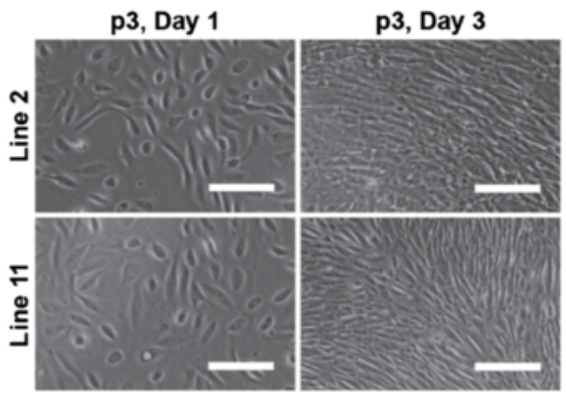

d

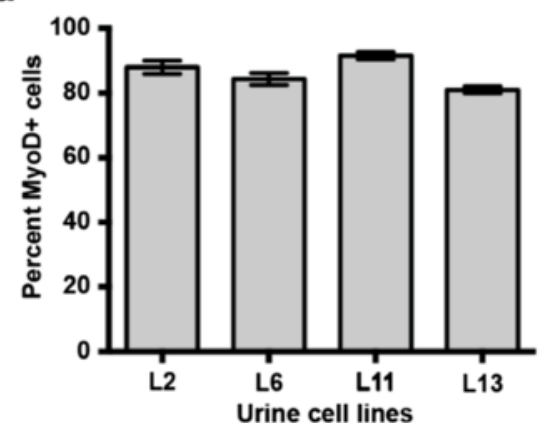

Fig. 1 Transduction of urine-derived cells (UDCs) with an iMyoD lentiviral construct for myogenic reprogramming. a General overview for generating in vitro model of human skeletal muscle using UDCs and iMyoD construct. The cells were first isolated from urine samples and then transduced with lentivirus encoding an inducible MyoD. The construct includes a 4-hydroxytamoxifen (tamoxifen, TAM) response element from the estrogen receptor (ERt) downstream of the basic helix loop helix (bHLH) DNA binding domain of MyoD. After induction with tamoxifen, cells were placed in differentiation media. b Bright-field images of isolated urine cell cultures at passage 3 . Scale bar $=25 \mu \mathrm{m}$. c Images of immunolabeled MyoD (red) in UDCs after transduction with the iMyoD lentivirus, and iMyoD-transduced urine cells treated with tamoxifen $(5 \mu \mathrm{M}, 48$ h). Nuclei were marked with Hoechst 33342 (blue). Scale bar= $100 \mu \mathrm{m}$. Inset images show increased MyoD localization to the nuclei after tamoxifen. Inset of tamoxifen-treated line 2 cells shows one nuclei with MyoD localization (left) and one without (right). Inset scale bar $=25 \mu \mathrm{m}$. $\mathbf{d}$ Lentiviral iMyoD transduction efficiency across four control urine cell lines, shown as the percent of MyoD-positive cells relative the total number of cells

form of skeletal myosin, was only detected when differentiation was extended to the $28 \mathrm{~d}$ time point (Fig. 3b). Similarly, real-time PCR detected most transcripts after 7 days of differentiation, with sustained or moderately increased expression over time (Fig. 3c). However, $D M D$ and $M Y H 2$ demonstrated greater temporal increases in transcript expression as myogenic maturation progressed (Fig. 3c). Evaluation of myotubes by immunofluorescence microscopy showed accumulation of $\alpha$-actinin, dystrophin, and fast myosin heavy chain (MHC), concordant with the messenger RNA (mRNA) data. Expression of these muscle proteins was associated with increased myotube formation, organization, and elongation, indicative of myogenic differentiation and maturation over time (Fig. 3d).

To further characterize iMyoD-directed myogenic maturation of urine cells, we analyzed reprogrammed cultures differentiated for 28 and 35 days. Lowmagnification bright-field images of tamoxifen-treated iMyoD lines 2 and 11 demonstrated elongated myotube formation after 28 days in HD differentiation media (Fig. 4a) in which expression of $\alpha$-actinin and dystrophin was observed throughout each fiber (Fig. 4a). Spontaneously twitching myotubes were observed in a subset of fields at both 28 and 35 days of culture, indicating sarcomere formation and function (Additional files 1 and 2: Movies S1 and S2). Sarcomere formation was confirmed with expression of fast myosin, $\alpha$-actinin, and titin, each localized internally to sarcolemmal dystrophin expression and in the expected pattern (Fig. 4b). These results demonstrated efficient myogenic reprogramming, differentiation, and maturation of iMyoD-transduced urine cells.

\section{Direct reprogramming of urine cells to model muscular dystrophy}

The iMyoD reprogramming strategy was applied to urine cells derived from two patients with Limb Girdle Muscular Dystrophy type 2C (LGMD2C), which results from loss of function mutations in the 


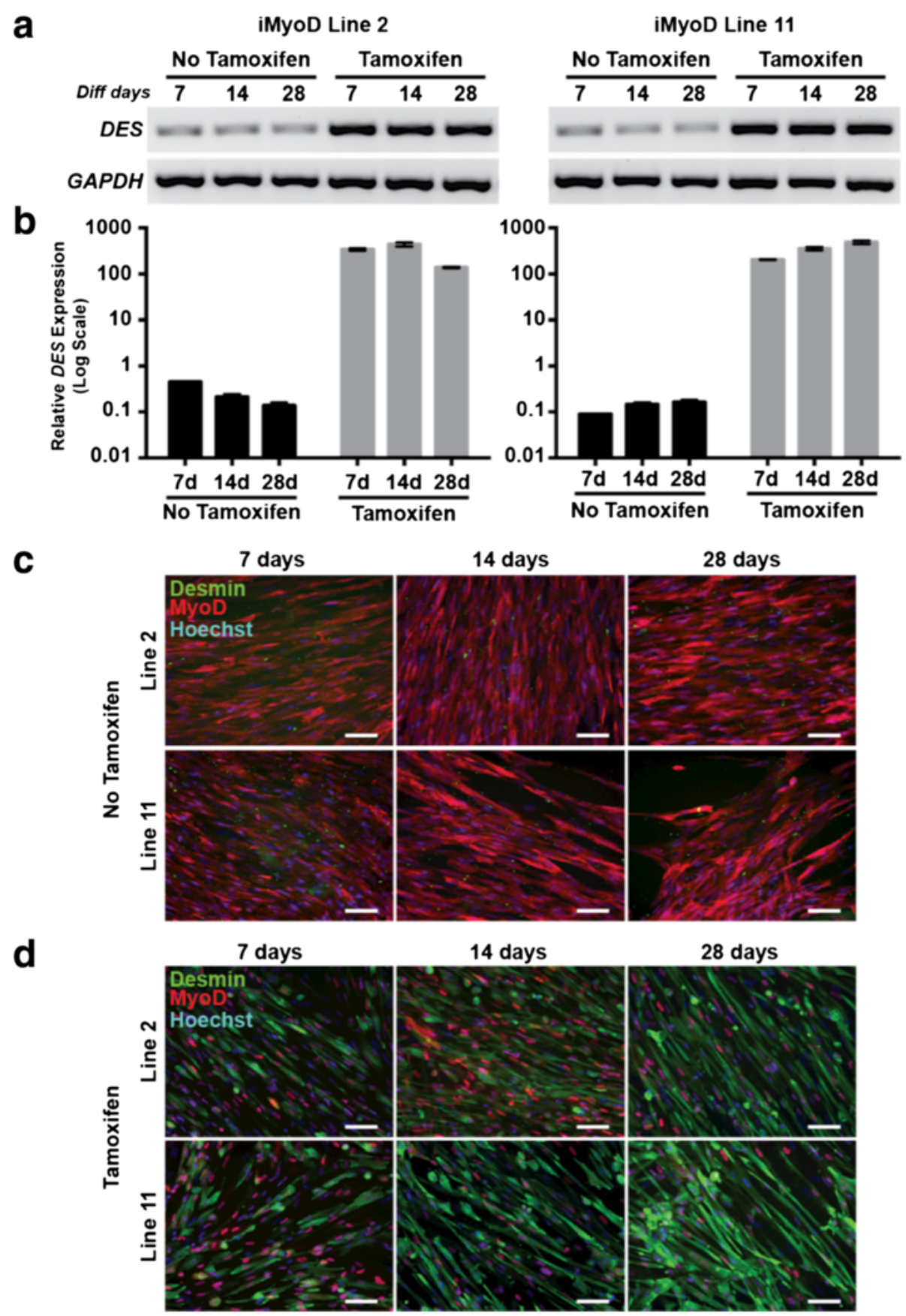

Fig. 2 Tamoxifen-induced myogenic reprogramming of iMyoD-transduced UDCs. a RT-PCR time course evaluation of DES (desmin) mRNA expression in two UDC lines (2 and 11). iMyoD-transduced cells were treated with or without tamoxifen $(2.5 \mu \mathrm{M}, 24 \mathrm{~h})$ and then cultured in differentiation media as indicated $(7,14$, and 28 days). GAPDH mRNA expression is shown as a control. b Real-time semi-quantitative PCR results for DES mRNA expression in urine cell lines 2 and 11. c Immunofluorescence microscopy of MyoD (red) and desmin (green) protein expression in iMyoD-transduced control lines 2 and 11, without tamoxifen treatment. In the uninduced state, MyoD demonstrated a cytoplasmic distribution, and no appreciable desmin expression was observed. Nuclei (blue); Scale bar $=100 \mu \mathrm{m}$. d After induction, MyoD (red) became more nuclear concomitant with cytoplasmic desmin (green) protein expression. Myotube elongation progressed over time in culture. Scale bar $=100 \mu \mathrm{m}$

gene encoding $\gamma$-sarcoglycan (SGCG) (Fig. 5a). Urinederived cells from each patient were successfully transduced with the $\mathrm{iMyoD}$ lentivirus. Real-time PCR analysis of tamoxifen-treated iMyoD LGMD2C cells demonstrated successful reprogramming into the myogenic lineage (Fig. 5b) associated with morphological myotube formation (Fig. 5c). RT-PCR analysis demonstrated full-length SGCG transcript 
a

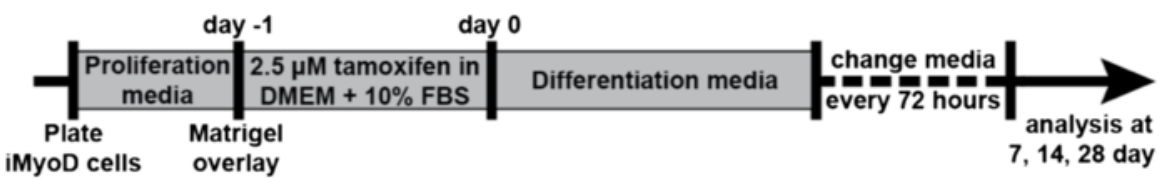

b

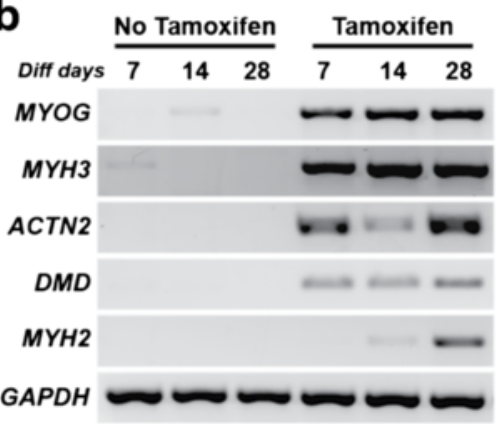

C

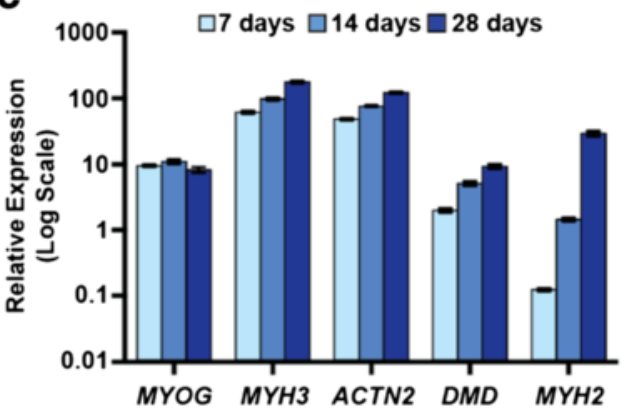

d
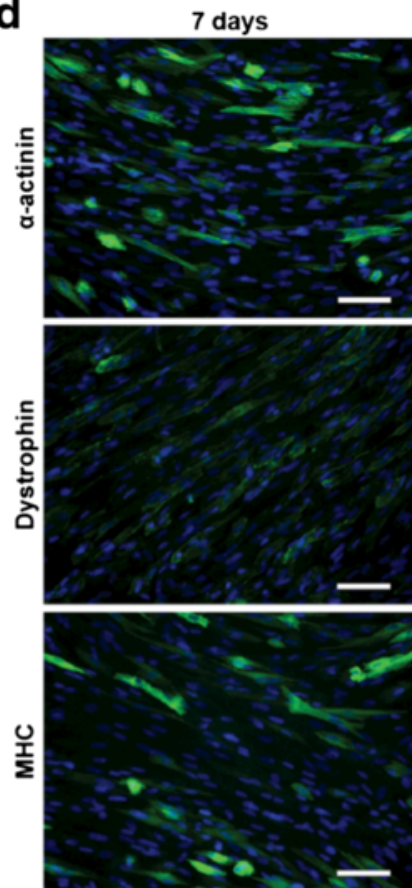

14 days
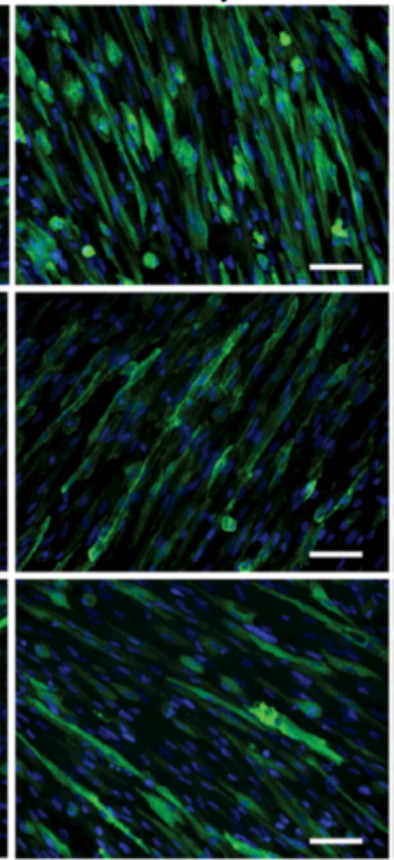

28 days
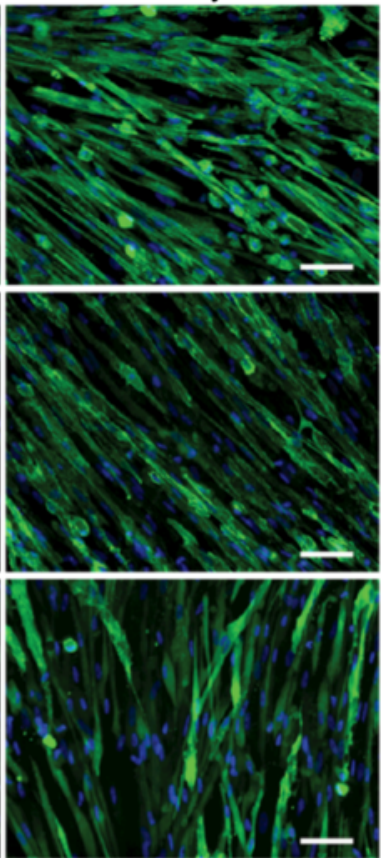

Fig. 3 Myogenic differentiation and maturation of induced iMyoD UDCs. a Timeline for differentiation of iMyoD-transduced cells. b RT-PCR for myogenic maturation markers in iMyoD-transduced line 2, with or without tamoxifen $(2.5 \mu \mathrm{M}, 24 \mathrm{~h})$, and culture in differentiation media as indicated (7, 14, and 28 days). Myogenin (MYOG) and embryonic myosin heavy chain (MYH3) were the earliest markers detected. $\mathbf{c}$ Real-time semi-quantitative PCR results demonstrated that dystrophin (DMD) and adult myosin heavy chain (MYH2) were detected with further differentiation. $\mathbf{d}$ Images of myogenic markers in iMyoD-transduced line 2. Increased muscle marker expression over time was accompanied by myotube elongation. Nuclei (blue); Scale bar $=100 \mu \mathrm{m}$

expression in reprogrammed control iMyoD cells and truncated mutant SGCG transcript expression in the reprogrammed iMyoD patient cells (Fig. 5a). Sarcolemmal associated $\gamma$-sarcoglycan protein was observed in control but not in LGMD2C cells, consistent with their mutation status (Fig. 5d).

We applied the same iMyoD strategy to DMD urine cells. The DMD patient harbored a frameshift deletion of exons 46 and 47 (ex46/47del) (Fig. 6a). RT-PCR analysis demonstrated full-length $D M D$ transcript expression in reprogrammed control iMyoD cells and truncated $D M D$ mutant transcript expression in the reprogrammed iMyoD patient cells (Fig. 6a). Sequence analysis of the PCR-amplified transcripts confirmed the deletion of exons 46 and 47 in the reprogrammed patient-derived cells (Fig. 6b). Sarcolemma-associated 


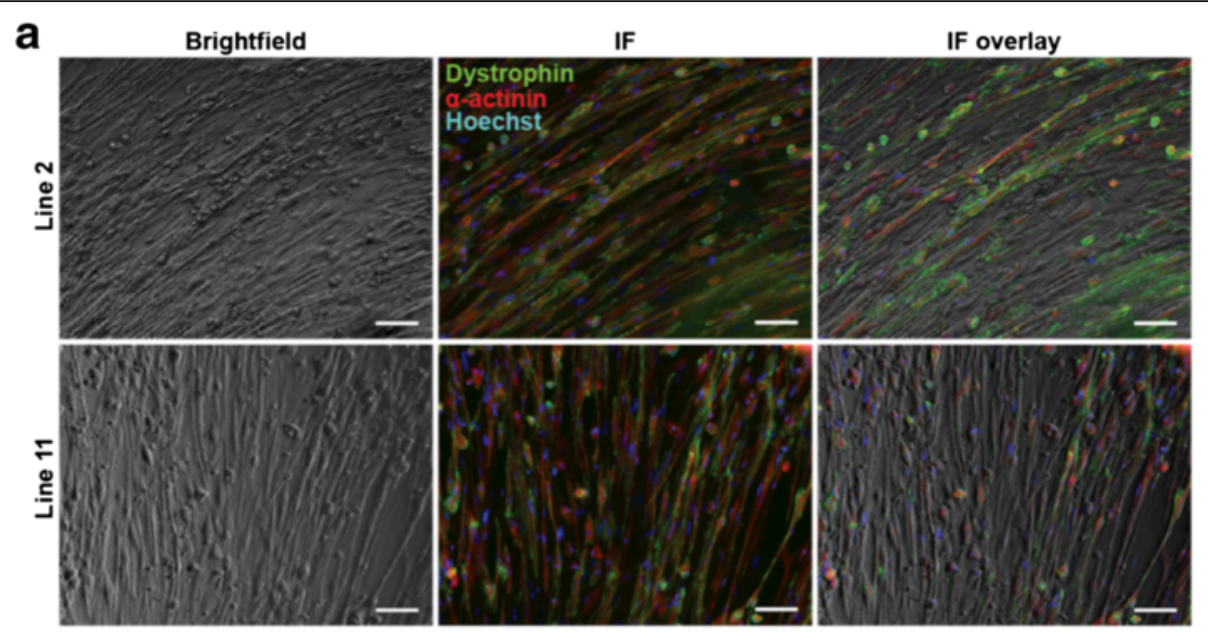

b
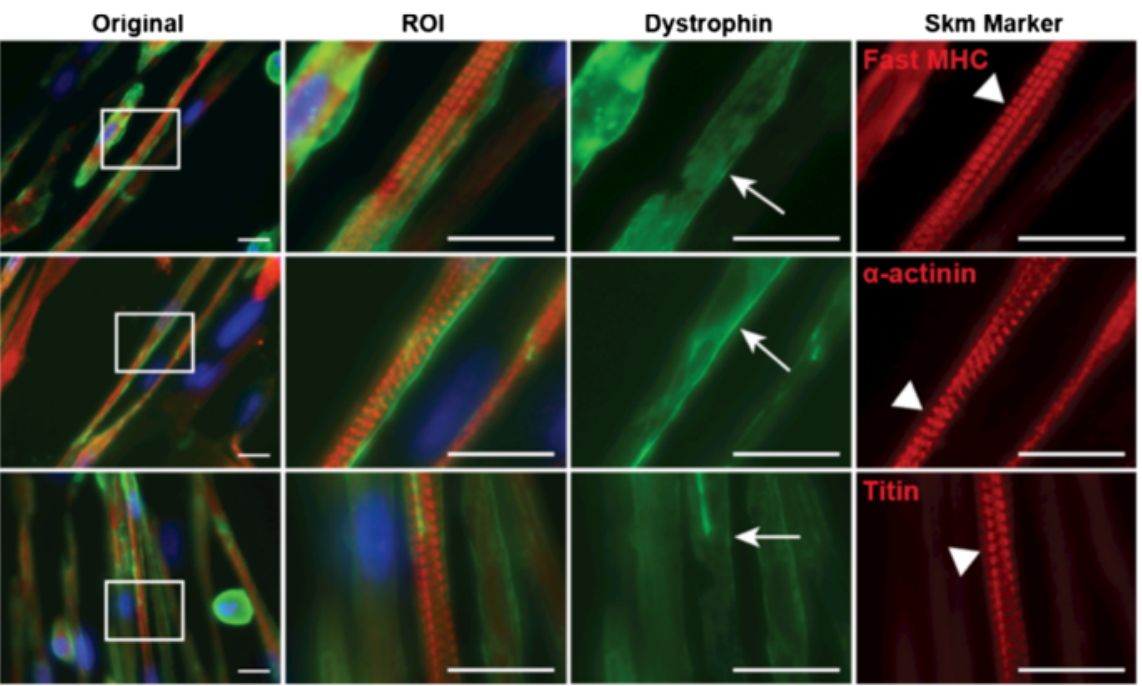

Fig. 4 Maturation of myotubes at 28-day and 35-day time points. a Bright-field images of myotube formation at the 28-day time point in iMyoD lines 2 and 11 after induction and differentiation (left panels). Middle panels show images of dystrophin (green) and a-actinin (red) protein expression, taken in the same field as the bright-field panels. The right panels show the merged images revealing dystrophin and a-actinin expressions throughout the lengths of the myotubes. Scale bar $=100 \mu \mathrm{m}$. b Images of iMyoD-induced UDCs after 35 days of differentiation showing expression of fast MHC, $\mathrm{a}$-actinin, and titin. The magnified region of interest (ROI) is outlined in the first panels (white rectangles). White arrows highlight the membrane localization of dystrophin (green). White arrowheads highlight the formation of sarcomeres (red). Scale bar $=25 \mu \mathrm{m}$

dystrophin protein was observed in control cells (Fig. 6c). In contrast, dystrophin protein was not detected in reprogrammed DMD patient cells after 28d differentiation (Fig. 6c). Thus, the iMyoD strategy in urine cells replicated the primary LGMD and DMD phenotypes in vitro.

\section{CRISPR/Cas9 mediated genome editing in single urine cell clones}

Finally, we evaluated CRISPR (clustered regularly interspaced short palindromic repeats)/Cas9 (CRISPR-associated protein 9) mediated genome editing in urine cells. For these studies, the goal was to introduce the most common LGMD2C disease-causing mutation into control cell lines by deleting one thymine from SGCG exon 6 to disrupt the reading frame [26]. To generate the $521 \Delta \mathrm{T}$ mutation, we used a gRNA targeting downstream of five sequential thymine residues and a single-stranded oligonucleotide (ssODN) to mediate homology-directed repair (HDR). The ssODN targeting the minus strand contained a synonymous $\mathrm{G}>\mathrm{A}$ transition to disrupt the protospacer adjacent motif (PAM) and introduce a novel Alu1 restriction site (Fig. 7a). CRISPR components were electroporated into control UDC lines 2 and 11, followed by FACS single cell sorting for GFP into 96-well plates. Approximately $20 \%$ of the 

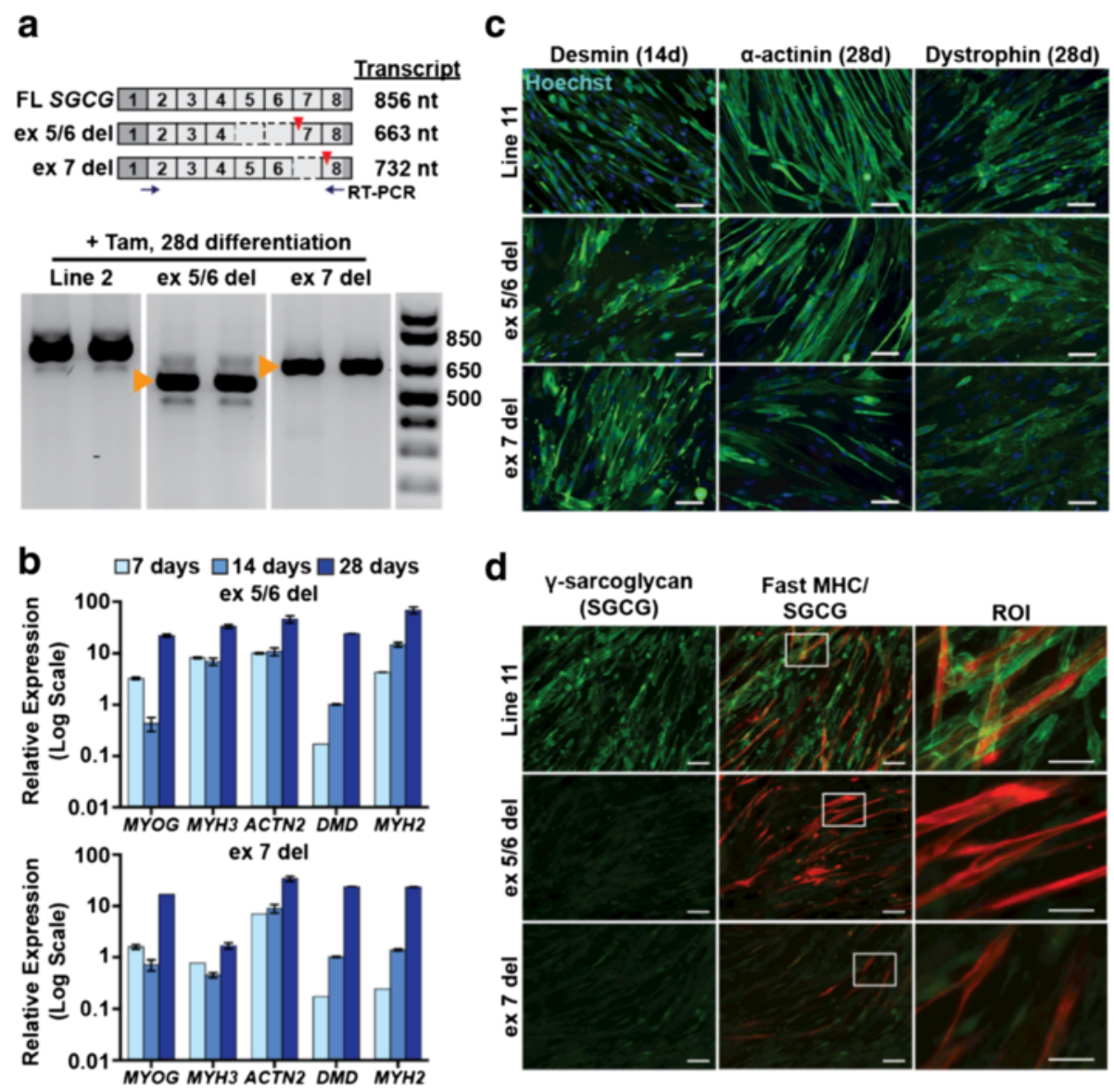

Fig. 5 Myogenic reprogramming of LGMD2C UDCs. a Schematic showing exon organization of the $\gamma$-sarcoglycan (SGCG) mRNA transcript and two LGMD2C patients with frameshifting deletions affecting the SGCG locus that lead to premature stop codons (red triangles). One has a deletion of exons 5 and 6 (ex5/6del), and the other has a deletion of exon 7 (ex7del). RT-PCR demonstrated SGCG transcripts from UDCs after treatment with tamoxifen $(2.5 \mu \mathrm{M}, 24 \mathrm{~h})$ and culture in differentiation media for 28d. Orange arrowheads indicate the mutant transcripts lacking the expected exons. $\mathbf{b}$ Real-time semi-quantitative PCR results demonstrate myogenic reprogramming of ex5/6del (top) and ex7del (bottom) after treatment with tamoxifen ( $2.5 \mu \mathrm{M}$, $24 \mathrm{~h}$ ) and culture in differentiation media as indicated (7, 14, and 28 days). c Images of myogenic markers in iMyoD control and LGMD2C ex5/6del and ex7del patient-derived lines. LGMD2C patient-derived cells formed myotubes comparable to those of control cells. Nuclei (blue); Scale bar $=50 \mu \mathrm{m}$. d Desmin, a-actinin, dystrophin, and fast MHC were expressed in LGMD2C cells. $\gamma$-sarcoglycan (green) was not detected. White boxes outline double-labeled regions of interest (ROI) enlarged in far right panels. Images show $\gamma$-sarcoglycan expression and localization to the membrane in line 11, as opposed to no detectable expression of $\gamma$-sarcoglycan in LGMD2C patient myotubes. Scale bar $=100 \mu \mathrm{m}$ in left and middle panels; $50 \mu \mathrm{m}$ in right panels

clones derived from single sorted cells grew sufficiently so that they could be evaluated for CRISPRmediated gene editing (Fig. 7b). The proliferation capacity of FACS-sorted clones was similar in untreated cells and cells electroporated with the Cas9 GFP plasmid alone.

The genomic region including SGCG exon 6 was PCR amplified to analyze the results of gene editing. Restriction digest of the PCR products revealed the presence of an Alu1 site in a subset of clones, indicative of HDR repair (Fig. 7c). Sequence analysis demonstrated efficient genome editing in UDCs with the expected variety of both heterozygous and homozygous mutations, including insertions and deletions from nonhomologous end joining (NHEJ) repair and specific point mutations directed by HDR (Fig. 7c, d). Generation of clones with both heterozygous and homozygous $521 \Delta \mathrm{T}$ mutations was evident in line 2 (Fig. 7c, d). Interestingly, a subset of HDR-edited clones contained a point mutation in the PAM region without the upstream thymine deletion, suggesting that this region is refractory to editing and/or required a gRNA target closer to the $5 \mathrm{~T}$ region. A summary of the gene editing results is shown in Fig. 7b, Additional file 3: Table S1 and S2, and Figure S1 and S2. Collectively, these data demonstrated the ability to 


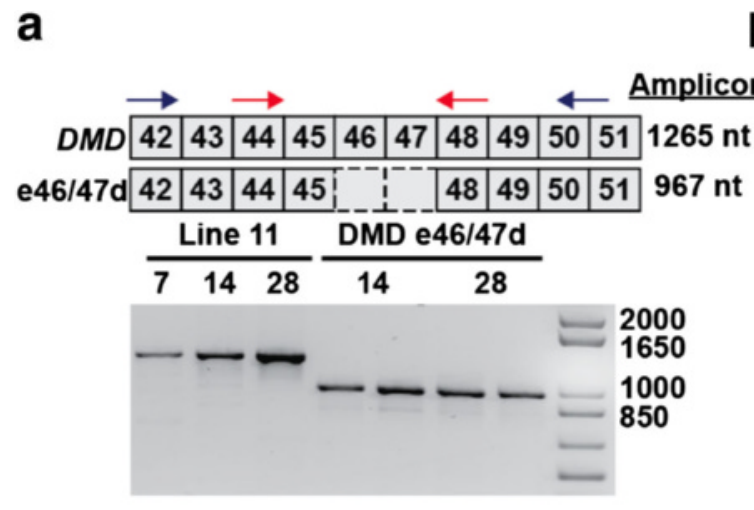

C
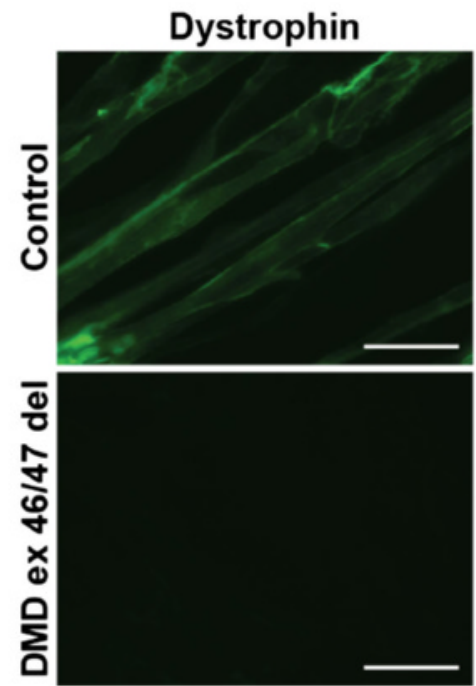

b

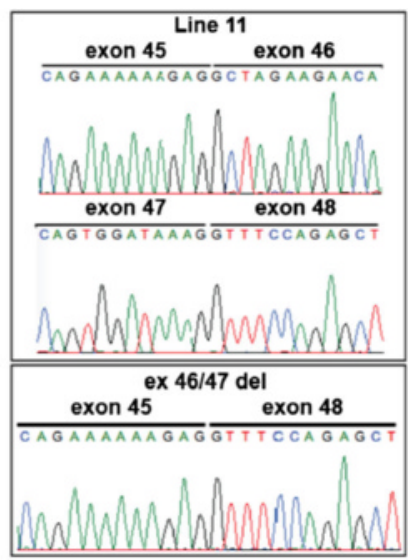

Merge
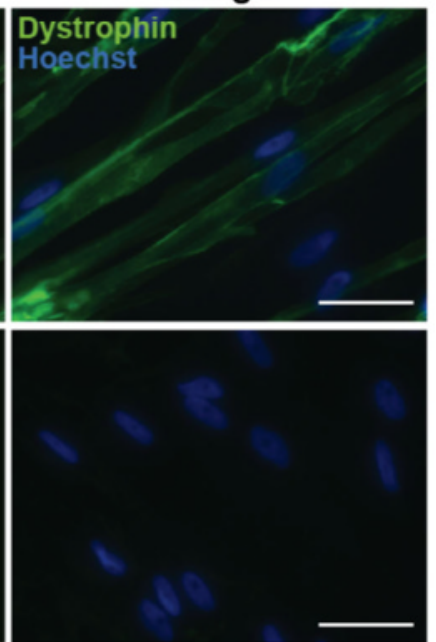

Fig. 6 Myogenic reprogramming of DMD patient-derived urine cells. a Schematic showing organization of dystrophin exons 42 to 51 (top) and DMD patient with a frameshift deletion of exons 46 and 47 (bottom). RT-PCR demonstrated DMD transcript expression from iMyoD-transduced line 11 control and DMD ex46/47del mutant cell lines, after tamoxifen induction $(2.5 \mu \mathrm{M}, 24 \mathrm{~h})$ and differentiation as indicated. Blue arrows indicate the location of the outer primers that produce the PCR products in the gel below. Red arrows indicate nested primers used to generate transcripts used for sequencing. b Sequencing results from RT-PCR products from iMyoD-reprogrammed urine-derived cells. The sequenced transcripts were generated from a set of nested primers (red) shown in a. Line 11 showed the expected exon45-exon46 and exon47-exon48 junctions. The ex46/47del DMD individual shows the out-of-frame mutant exon45-exon48 junction. c Immunofluorescence images of iMyoD line 17 and DMD ex46/47del cells labeled with dystrophin using the NCL-DYSB antibody to dystrophin. The antibody to dystrophin showed membrane localization of dystrophin in control cells (top). No dystrophin was detected in DMD patient-derived myotubes. Scale bar $=50 \mu \mathrm{m}$

edit the genome of urine-derived cells with CRISPR/ Cas9, generating single cell clones with a specific point mutation.

\section{Discussion}

The limited availability of human skeletal muscle tissue encumbers the study of muscle development and the pathology of muscle disease. Many novel strategies have been taken to overcome this limitation, relying on genetic engineering with direct and indirect reprogramming of cells. Regardless of strategy, a key component to success is the initial cell source, specifically the ease of acquisition and ability to reprogram. Urine is an underutilized, noninvasive, and cost effective source of primary human cells that has served as a platform for urothelial-based culture and iPSC reprogramming [19-21]. Recent advances in urine cell isolation have generated clonal populations of autologous human cells with a high proliferative capacity, evident telomerase activity, and plasticity for reprogramming into multiple lineages $[20,21]$. These characteristics make urine-derived cells an attractive source of primary human cells for in vitro models. We now extended these findings showing that inducible expression of MyoD efficiently reprogrammed UDCs into the myogenic lineage, providing a simple strategy to 


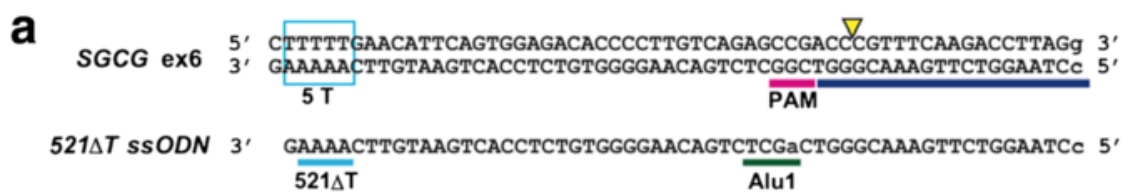

\section{b}

Efficacy of isolation and mutagenesis
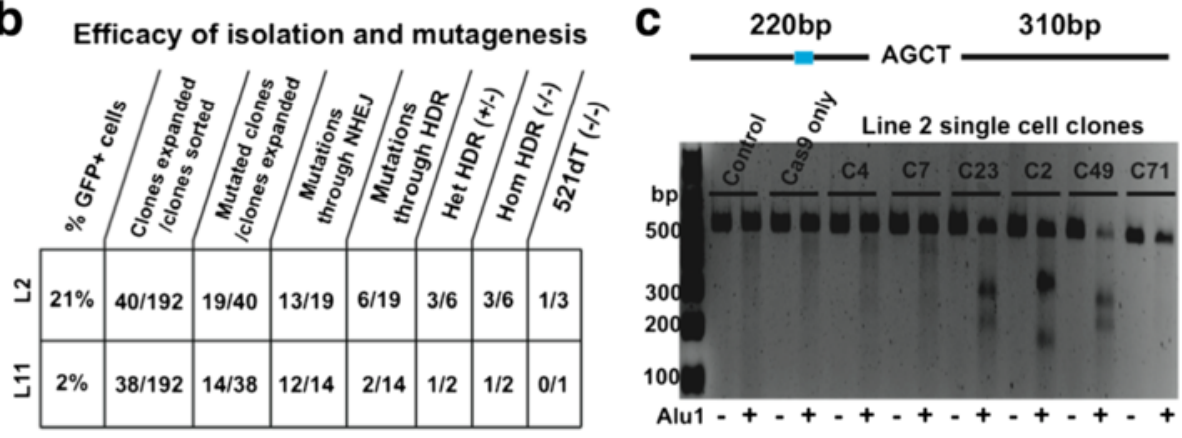

\section{d}

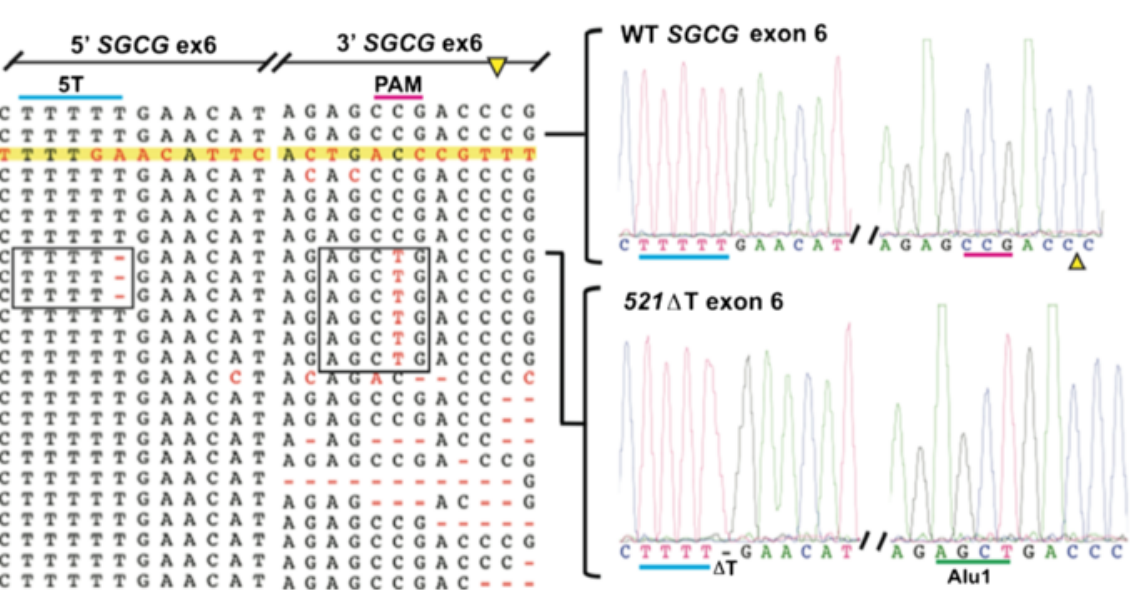

Fig. 7 CRISPR/Cas9-mediated gene editing in UDCs. a Targeting strategy to introduce the 521 $\Delta$ T point mutation into control SGCG exon 6 through gRNA guided, Cas9 nuclease generated double strand DNA break (DSB) with ssODN-mediated HDR repair. A portion of the SGCG exon 6 coding region is shown; a single $T$ was removed from the $5 T$ sequence region (cyan box). The downstream gRNA target sequence is highlighted in blue, the PAM sequence in pink, and the Cas9 DSB indicated by a yellow triangle. Below it is a portion of the 142 bp ssODN HDR repair template encoding the single $T$ deletion along with a downstream $\mathrm{G}>\mathrm{A}$ synonymous mutation (green) that destroys the PAM sequence and introduces an Alu1 restriction site. b Summary of efficiency of CRISPR/Cas9 protocol. c Alu1 restriction digest of a PCR-amplified genomic region including SGCG exon 6 and the flanking intronic sequences. $\mathbf{d}$ Left, partial summary of the Sanger sequencing results from individual line 2 edited clones. Right, the DNA trace from an unedited control and a clone with a homozygous deletion of a single T from the 5T of SGCG exon 6, recreating the most common LGMD2C mutation

generate an in vitro model of skeletal muscle from virtually any human subject.

Urine cells demonstrate an inclination towards the mesodermal lineage. A subpopulation of cells, classified as urine stem cells, express pericyte and mesenchymal stem cell markers and were able to differentiate into podocytes, smooth muscle, and urothelial cells [20, 21, 32]. The ability of UDCs to differentiate into skeletal muscle is less defined. Studies showed growth on specific biomaterials, presence of specific growth factors, or defined media modifications promoted expression of myogenic markers such as MyoD, desmin, and myosin [23, 34].
However, these cells were not observed to form striations nor to display contractile properties. Given the known expression of these markers, it is possible that without sufficient MyoD, UDCs differentiate more towards smooth rather than striated muscle lineages [21]. The strategy with an induced MyoD pulse combined with media manipulation drove urine cells through the initial stages of myogenic development into a more mature phenotype, complete with expression of dystrophin, adult myosin, titin, and $\gamma$-sarcoglycan, along with the presence of functional sarcomeres. Despite these advancements, this cellular model still lacks major 
components of mature muscle such as transverse tubules and resident stem cell niches.

An alternative strategy to direct reprogramming with MyoD would be to first reprogram UDCs into iPSCs and then use the iPSC lines for myogenic differentiation. This approach has the advantage of iPSC self-renewal, although immortalization of UDCs directly could obviate this need in vitro. DMD UDCs were previously successfully reprogrammed into iPSC and differentiated into cardiomyocytes, recapitulating cardiac phenotypes in vitro [4].

Skeletal muscle differentiation of iPSCs has proven more challenging, although more efficient skeletal muscle differentiation from iPSC using small molecules has recently been reported [7]. Interestingly, the ease of urine cell isolation, transduction with iMyoD virus, and reprogramming into iPSC suggest an ability to employ multiple parallel models for the study of muscle disease. A summary of cell types for modeling muscle disease and the timeline for generating these models in culture is shown in Fig. 8.

We also showed that UDCs can be directly gene edited using CRISPR/Cas9 technology. The use of gene editing for muscle disease has focused intently on genetic correction strategies with significant success on the study of muscular dystrophy, as recent studies showed the ability to correct DMD disease-causing mutations in animal models and human cells [35-43]. However, the ability to insert mutations of interest is also useful since it allows the study of disease models without the need to access patients directly. Nevertheless, gene editing in human iPSCs is challenged by the availability of patient cell lines, isolation of pure clonal populations, and low editing efficiency, especially when targeting precise mutations with homology-dependent repair [29, 44, 45]. While some technical aspects of the CRISPR/Cas9 system are still undergoing improvement, the relative ease of urine cell isolation, coupled with the ability to generate single cell clones, makes this an attractive primary cell platform for CRISPR/Cas9 gene editing.

\section{Conclusions}

Here, we showed that direct reprogramming of urine cells using MyoD is feasible from patients with muscle disease and can recapitulate the basic disease phenotype.

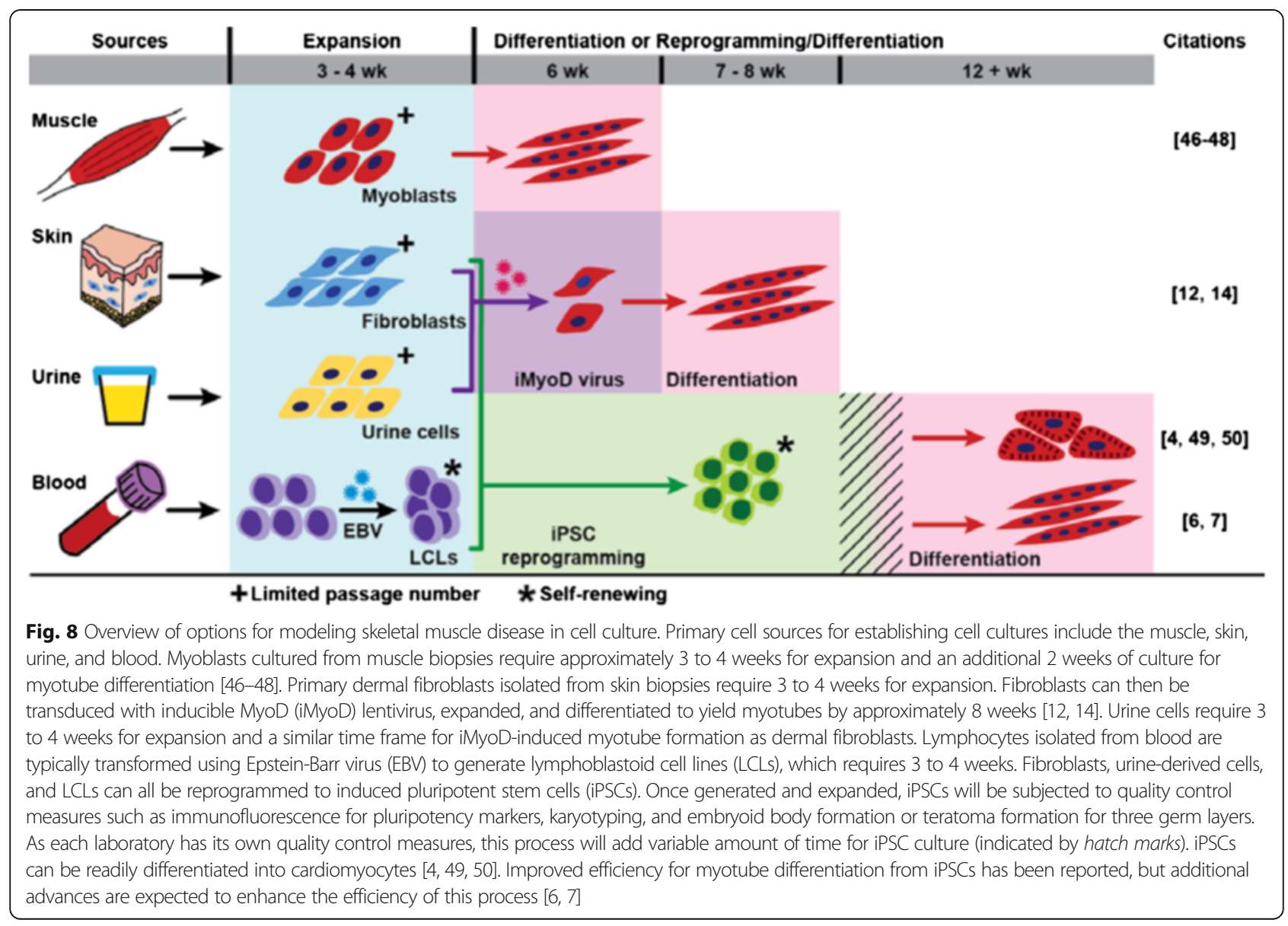


Because urine cells are readily available and collected noninvasively, this approach provides a highly useful model of human muscle disease.

\section{Additional files}

Additional file 1: Movie S1. Video showing spontaneous myotube twitching.

Additional file 2: Movie S2. Video showing spontaneous myotube twitching.

Additional file 3: Table S1. Sequencing results for line 2 clones. Table S2. Sequencing results for line 11 clones. Figure S1. Example of sequencing results from CRISPR/Cas9 edited urine line 2. Figure S2. Example of sequencing results from CRISPR/Cas9 edited urine line 11. (PDF $1694 \mathrm{~kb}$ )

\section{Abbreviations}

UDCs: Urine-derived cells; iMyoD: Inducible MyoD; FBS: Fetal bovine serum; DMEM: Dulbecco's Modified Eagle Medium; PBS: Phosphate-buffered saline; FGF: Fibroblast growth factor; PDGF: Platelet-derived growth factor; EGF: Epidermal growth factor; RT-PCR: Reverse transcription polymerase chain reaction; CRISPR: Clustered regularly interspaced short palindromic repeats; Cas9: CRISPR-associated protein 9; ssODN: Single-stranded oligodeoxynucleotide; PAM: Protospacer adjacent motif; gRNA: Guide RNA; HDR: Homology-dependent repair; NHEJ: Nonhomologous end joining; DSB: Double strand DNA break; FACS: Fluorescence-activated cells sorting; RFLP: Restriction fragment length polymorphism; LGMD: Limb girdle muscular dystrophy; DMD: Duchenne muscular dystrophy; SGCG: $\gamma^{-}$ Sarcoglycan; MHC: Myosin heavy chain; iPSCs: Induced pluripotent stem cells; SEM: Standard error of the mean; HD: Hydrocortisone dexamethasone

\section{Acknowledgements}

We thank the patients for their participation and the Kurt+Peter Foundation. We acknowledge the Skin Disease Research Center, Flow Cytometry Core, and NUSeq Core Facilities at Northwestern University.

\section{Funding}

This work was supported by NIH HL61322, NIH T32 HD009007, and the Kurt +Peter Foundation.

\section{Authors' contributions}

EYK conducted the experiments, analyzed the data, prepared the figures, and wrote the manuscript; PP conducted the experiments; LDC coordinated the collection of human materials; EMM analyzed the data and wrote and edited the manuscript; EW conducted the experiments, analyzed the data, prepared the figures, and wrote the manuscript. All authors read and approved the final manuscript.

\section{Competing interests}

The authors have no competing financial interests related to the conduct of the work presented in this manuscript.

\section{Ethics approval and consent to participate}

Written and informed consent was obtained from all human subjects. All work was approved by and conducted under the Institutional Review Boards of the University of Chicago (IRB8249) and Northwestern University (IRB STU00104548), which serve as the ethics boards for each institution, respectively. All studies were conducted in compliance with the Helsinki Declaration.

\section{Author details}

${ }^{1}$ Molecular Pathogenesis and Molecular Medicine, The University of Chicago, Chicago, USA. ${ }^{2}$ Center for Genetic Medicine, Northwestern University Feinberg School of Medicine, 303 E. Superior St., Chicago, IL 60611, USA.

Received: 18 May 2016 Accepted: 23 August 2016

Published online: 15 September 2016

\section{References}

1. Mercuri E, Muntoni F. Muscular dystrophies. Lancet. 2013;381(9869):845-60.

2. Rahimov F, Kunkel LM. The cell biology of disease: cellular and molecular mechanisms underlying muscular dystrophy. J Cell Biol. 2013;201(4):499-510.

3. Lin B, Li Y, Han L, Kaplan AD, Ao Y, Kalra S, Bett GC, Rasmusson RL, Denning C, Yang L. Modeling and study of the mechanism of dilated cardiomyopathy using induced pluripotent stem cells derived from individuals with Duchenne muscular dystrophy. Dis Model Mech. 2015;8(5):457-66.

4. Guan X, Mack DL, Moreno CM, Strande JL, Mathieu J, Shi Y, Markert CD, Wang Z, Liu G, Lawlor MW, et al. Dystrophin-deficient cardiomyocytes derived from human urine: new biologic reagents for drug discovery. Stem Cell Res. 2014;12(2):467-80.

5. Dick E, Kalra S, Anderson D, George V, Ritso M, Laval SH, Barresi R, Aartsma-Rus A, Lochmuller H, Denning C. Exon skipping and gene transfer restore dystrophin expression in human induced pluripotent stem cells-cardiomyocytes harboring DMD mutations. Stem Cells Dev. 2013;22(20):2714-24.

6. Darabi R, Perlingeiro RC. Derivation of skeletal myogenic precursors from human pluripotent stem cells using conditional expression of PAX7. Methods Mol Biol. 2016:1357:423-39.

7. Chal J, Oginuma M, Al Tanoury Z, Gobert B, Sumara O, Hick A, Bousson F, Zidouni $Y$, Mursch $C$, Moncuquet $P$, et al. Differentiation of pluripotent stem cells to muscle fiber to model Duchenne muscular dystrophy. Nat Biotechnol. 2015:33(9):962-9.

8. Portier GL, Benders AG, Oosterhof A, Veerkamp JH, van Kuppevelt TH. Differentiation markers of mouse $\mathrm{C} 2 \mathrm{C} 12$ and rat $\mathrm{L} 6$ myogenic cell lines and the effect of the differentiation medium. In Vitro Cell Dev Biol Anim. 1999;35(4):219-27.

9. Owens J, Moreira K, Bain G. Characterization of primary human skeletal muscle cells from multiple commercial sources. In Vitro Cell Dev Biol Anim. 2013;49(9):695-705.

10. Davis $R L$, Weintraub $H$, Lassar AB. Expression of a single transfected cDNA converts fibroblasts to myoblasts. Cell. 1987;51(6):987-1000

11. Tapscott SJ, Davis RL, Thayer MJ, Cheng PF, Weintraub H, Lassar AB. MyoD1: a nuclear phosphoprotein requiring a Myc homology region to convert fibroblasts to myoblasts. Science. 1988;242(4877):405-11.

12. Lattanzi L, Salvatori G, Coletta M, Sonnino C, Cusella De Angelis MG, Gioglio L, Murry CE, Kelly R, Ferrari G, Molinaro M, et al. High efficiency myogenic conversion of human fibroblasts by adenoviral vector-mediated MyoD gene transfer. An alternative strategy for ex vivo gene therapy of primary myopathies. J Clin Invest. 1998;101(10):2119-28.

13. Cooper ST, Kizana E, Yates JD, Lo HP, Yang N, Wu ZH, Alexander IE, North $\mathrm{KN}$. Dystrophinopathy carrier determination and detection of protein deficiencies in muscular dystrophy using lentiviral MyoD-forced myogenesis. Neuromuscul Disord. 2007;17(4):276-84.

14. Kimura E, Han JJ, Li S, Fall B, Ra J, Haraguchi M, Tapscott SJ, Chamberlain JS. Cell-lineage regulated myogenesis for dystrophin replacement: a novel therapeutic approach for treatment of muscular dystrophy. Hum Mol Genet. 2008:17(16):2507-17.

15. Kendall GC, Mokhonova El, Moran M, Sejbuk NE, Wang DW, Silva O, Wang RT, Martinez L, Lu QL, Damoiseaux R, et al. Dantrolene enhances antisense-mediated exon skipping in human and mouse models of Duchenne muscular dystrophy. Sci Transl Med. 2012:4(164):164ra160.

16. Hollenberg SM, Cheng PF, Weintraub H. Use of a conditional MyoD transcription factor in studies of MyoD trans-activation and muscle determination. Proc Natl Acad Sci U S A. 1993;90(17):8028-32.

17. Ingelfinger JR. Nephrogenic adenomas as renal tubular outposts. N Engl J Med. 2002;347(9):684-6.

18. Rahmoune $\mathrm{H}$, Thompson PW, Ward JM, Smith CD, Hong G, Brown J. Glucose transporters in human renal proximal tubular cells isolated from the urine of patients with non-insulin-dependent diabetes. Diabetes. 2005;54(12):3427-34.

19. Dorrenhaus A, Muller JI, Golka K, Jedrusik P, Schulze H, Follmann W. Cultures of exfoliated epithelial cells from different locations of the human urinary tract and the renal tubular system. Arch Toxicol. 2000;74(10):618-26.

20. Bharadwaj S, Liu G, Shi Y, Wu R, Yang B, He T, Fan Y, Lu X, Zhou X, Liu H, et al. Multipotential differentiation of human urine-derived stem cells: potential for therapeutic applications in urology. Stem Cells. 2013;31(9):1840-56.

21. Zhang $Y$, McNeill E, Tian H, Soker S, Andersson KE, Yoo JJ, Atala A. Urine derived cells are a potential source for urological tissue reconstruction. J Urol. 2008;180(5):2226-33.

22. Afzal MZ, Strande JL. Generation of induced pluripotent stem cells from muscular dystrophy patients: efficient integration-free reprogramming of urine derived cells. J Vis Exp. 2015;95:52032. 
23. Chen W, Xie M, Yang B, Bharadwaj S, Song L, Liu G, Yi S, Ye G, Atala A, Zhang Y. Skeletal myogenic differentiation of human urine-derived cells as a potential source for skeletal muscle regeneration. J Tissue Eng Regen Med. 2014.

24. Zhou T, Benda C, Dunzinger S, Huang Y, Ho JC, Yang J, Wang Y, Zhang Y, Zhuang Q, Li Y, et al. Generation of human induced pluripotent stem cells from urine samples. Nat Protoc. 2012;7(12):2080-9.

25. Zuk PA, Zhu M, Ashjian P, De Ugarte DA, Huang Jl, Mizuno H, Alfonso ZC, Fraser JK, Benhaim P, Hedrick MH. Human adipose tissue is a source of multipotent stem cells. Mol Biol Cell. 2002;13(12):4279-95.

26. McNally EM, Passos-Bueno MR, Bonnemann CG, Vainzof M, de Sa ME, Lidov HG, Othmane KB, Denton PH, Vance JM, Zatz M, et al. Mild and severe muscular dystrophy caused by a single gamma-sarcoglycan mutation. Am J Hum Genet. 1996:59(5):1040-7.

27. Ran FA, Hsu PD, Wright J, Agarwala V, Scott DA, Zhang F. Genome engineering using the CRISPR-Cas9 system. Nat Protoc. 2013;8(11):2281-308.

28. Mali P, Yang L, Esvelt KM, Aach J, Guell M, DiCarlo JE, Norville JE, Church GM. RNA-guided human genome engineering via Cas9. Science. 2013; 339(6121):823-6.

29. Chen F, Pruett-Miller SM, Huang Y, Gjoka M, Duda K, Taunton J, Collingwood TN, Frodin M, Davis GD. High-frequency genome editing using ssDNA oligonucleotides with zinc-finger nucleases. Nat Methods. 2011:8(9):753-5.

30. Zhou T, Benda C, Duzinger S, Huang Y, Li X, Li Y, Guo X, Cao G, Chen S, Hao $L$, et al. Generation of induced pluripotent stem cells from urine. J Am Soc Nephrol. 2011;22(7):1221-8.

31. Felix JS, Sun TT, Littlefield JW. Human epithelial cells cultured from urine: growth properties and keratin staining. In Vitro. 1980;16(10):866-74.

32. Bharadwaj S, Liu G, Shi Y, Markert C, Andersson KE, Atala A, Zhang Y. Characterization of urine-derived stem cells obtained from upper urinary tract for use in cell-based urological tissue engineering. Tissue Eng Part A. 2011;17(15-16):2123-32.

33. Berkes CA, Tapscott SJ. MyoD and the transcriptional control of myogenesis. Semin Cell Dev Biol. 2005;16(4-5):585-95.

34. Liu G, Wang X, Sun X, Deng C, Atala A, Zhang Y. The effect of urine-derived stem cells expressing VEGF loaded in collagen hydrogels on myogenesis and innervation following after subcutaneous implantation in nude mice. Biomaterials. 2013;34(34):8617-29.

35. Li HL, Fujimoto N, Sasakawa N, Shirai S, Ohkame T, Sakuma T, Tanaka M, Amano N, Watanabe A, Sakurai $H$, et al. Precise correction of the dystrophin gene in duchenne muscular dystrophy patient induced pluripotent stem cells by TALEN and CRISPR-Cas9. Stem Cell Rep. 2015;4(1):143-54.

36. Long C, MCAnally JR, Shelton JM, Mireault AA, Bassel-Duby R, Olson EN. Prevention of muscular dystrophy in mice by CRISPR/Cas9-mediated editing of germline DNA. Science. 2014;345(6201):1184-8.

37. Long C, Amoasii L, Mireault AA, McAnally JR, Li H, Sanchez-Ortiz E, Bhattacharyya S, Shelton JM, Bassel-Duby R, Olson EN. Postnatal genome editing partially restores dystrophin expression in a mouse model of muscular dystrophy. Science. 2016:351(6271):400-3.

38. Nelson CE, Hakim CH, Ousterout DG, Thakore PI, Moreb EA, Castellanos Rivera RM, Madhavan S, Pan X, Ran FA, Yan WX, et al. In vivo genome editing improves muscle function in a mouse model of Duchenne muscular dystrophy. Science. 2016;351(6271):403-7.

39. Ousterout DG, Kabadi AM, Thakore PI, Majoros WH, Reddy TE, Gersbach CA. Multiplex CRISPR/Cas9-based genome editing for correction of dystrophin mutations that cause Duchenne muscular dystrophy. Nat Commun. 2015;6:6244.

40. Tabebordbar M, Zhu K, Cheng JK, Chew WL, Widrick JJ, Yan WX, Maesner C, Wu EY, Xiao R, Ran FA, et al. In vivo gene editing in dystrophic mouse muscle and muscle stem cells. Science. 2016;351(6271):407-11.

41. Wojtal D, Kemaladewi DU, Malam Z, Abdullah S, Wong TW, Hyatt E, Baghestani Z, Pereira S, Stavropoulos J, Mouly V, et al. Spell checking nature: versatility of CRISPR/Cas9 for developing treatments for inherited disorders. Am J Hum Genet. 2016;98(1):90-101.

42. Xu L, Park KH, Zhao L, Xu J, El Refaey M, Gao Y, Zhu H, Ma J, Han R. CRISPRmediated genome editing restores dystrophin expression and function in mdx mice. Mol Ther. 2016;24(3):564-9.

43. Young CS, Hicks MR, Ermolova NV, Nakano H, Jan M, Younesi S, Karumbayaram S, Kumagai-Cresse C, Wang D, Zack JA, et al. A single CRISPR-Cas9 deletion strategy that targets the majority of DMD patients restores dystrophin function in hiPSC-derived muscle cells. Cell Stem Cell. 2016;18(4):533-40.

44. Yang L, Guell M, Byrne S, Yang JL, De Los Angeles A, Mali P, Aach J, KimKiselak C, Briggs AW, Rios X, et al. Optimization of scarless human stem cell genome editing. Nucleic Acids Res. 2013;41(19):9049-61.
45. Bialk P, Rivera-Torres N, Strouse B, Kmiec EB. Regulation of gene editing activity directed by single-stranded oligonucleotides and CRISPR/Cas9 systems. PLoS One. 2015;10(6):e0129308.

46. Rando TA, Blau HM. Primary mouse myoblast purification, characterization, and transplantation for cell-mediated gene therapy. J Cell Biol. 1994;125(6):1275-87.

47. Blau HM, Webster C. Isolation and characterization of human muscle cells. Proc Natl Acad Sci U S A. 1981;78(9):5623-7.

48. Cheng CS, El-Abd Y, Bui K, Hyun YE, Hughes RH, Kraus WE, Truskey GA. Conditions that promote primary human skeletal myoblast culture and muscle differentiation in vitro. Am J Physiol Cell Physiol. 2014;306(4):C385-395.

49. Lian X, Zhang J, Azarin SM, Zhu K, Hazeltine LB, Bao X, Hsiao C, Kamp TJ, Palecek SP. Directed cardiomyocyte differentiation from human pluripotent stem cells by modulating Wnt/beta-catenin signaling under fully defined conditions. Nat Protoc. 2013;8(1):162-75.

50. Burridge PW, Matsa E, Shukla P, Lin ZC, Churko JM, Ebert AD, Lan F, Diecke S, Huber B, Mordwinkin NM, et al. Chemically defined generation of human cardiomyocytes. Nat Methods. 2014;11(8):855-60.

\section{Submit your next manuscript to BioMed Central and we will help you at every step:}

- We accept pre-submission inquiries

- Our selector tool helps you to find the most relevant journal

- We provide round the clock customer support

- Convenient online submission

- Thorough peer review

- Inclusion in PubMed and all major indexing services

- Maximum visibility for your research

Submit your manuscript at www.biomedcentral.com/submit

) Biomed Central 